\title{
Administration of the BCG vaccination using the multipuncture method in schoolchildren: a comparison with the intradermal method
}

\author{
N Al Jarad, D W Empey, G Duckworth
}

\begin{abstract}
Background-BCG vaccination using the multipuncture device (the Heaf gun) is recommended in the $U K$ for infants and very small children only. The aim of this study was to investigate the rate of conversion of the tuberculin test, the safety and acceptability of BCG vaccination using the multipuncture device and to compare it with the conventional intradermal method in schoolchildren.

Methods-Schoolchildren attending schools in Tower Hamlets who were eligible for BCG vaccination were tuberculin tested using the Heaf gun. Those with grade $0-1$ reaction were randomised to receive BCG vaccination using either the multipuncture or the intradermal method. The site of BCG vaccination was inspected after eight weeks for inflammatory changes and scarring. A questionnaire about pain and inflammation at the site of vaccination was completed. The Heaf test was repeated at eight weeks and its results were assessed by an examiner unaware of the results of the previous Heaf test and the method of BCG administration. The Heaf test conversion was deemed to have occurred if there was a change of at least one grade in the response.
\end{abstract}

Results-One hundred and sixty nine children ( 83 girls) of mean age 11.8 years completed the study, of which 81 received BCG by the multipuncture method. The Heaf test did not convert in 22 of 81 (27.2\%) receiving BCG by the multipuncture device compared with six of $88(6.8 \%)$ who received the vaccine by the intradermal method (odds ratio $0.2,95 \%$ confidence interval 0.07 to 0.55 ). The BCG scar was visible in all children who had intradermal BCG compared with 67 of 81 $(81.8 \%)$ of the multipuncture group. The multipuncture method was less painful and caused fewer inflammatory changes than the intradermal method.

Conclusions-In schoolchildren the multipuncture device for administering BCG caused a lower rate of tuberculin conversion as measured by the Heaf test and less of an inflammatory response than the intradermal method. The method needs to be modified before it is applied on a wider scale to schoolchildren.

(Thorax 1999;54:762-764)

Keywords: BCG vaccination; multipuncture administration; tuberculosis
The value of BCG vaccination in schoolchildren in the UK in the prevention of tuberculosis has been sufficiently substantiated ${ }^{1-4}$ and it is now a routine clinical practice. The method of administration currently used is the intradermal method. This method is cheap and reliable, but it requires training and skill and is viewed with fear and anxiety by many children. The multipuncture device using a reusable gun with disposable head with 18 needles (Bignal Surgical Instruments, UK) is easy to use, requires little training and skill, is less painful, and is therefore more acceptable to children. This method has been validated and found to produce acceptable and reproducible results in small children ${ }^{5}$ and was recommended by the Department of Health for infants and very small children. ${ }^{6}$ It is widely used for infants for neonatal BCG whilst the Heaf test using this device is acceptable by older children. Previous studies with the multipuncture device have consistently found its efficacy to be inferior to the intradermal method of administration as judged by conversion of the tuberculin test. ${ }^{7-10}$

The East London and the City Health Authority has some of the highest rates of tuberculosis in the country and a high proportion of its population derives from countries where tuberculosis is still highly prevalent. It also has a very mobile population. BCG immunisation in school had been traditionally carried out by community paediatricians and suitably trained nurses. Given the scarcity of this resource in some areas and the difficulty in ensuring several medical visits to a school to immunise children who were not present on the first occasion, it was decided to explore BCG immunisation by the multipuncture method. Its main advantages were that it would not require administration by a medical practitioner and would be more acceptable to this particularly vulnerable group, thus resulting in less truancy and therefore better uptake on the immunisation day. With the above factors in mind, the Public Health Department of the East London and the City Health Authority and the local general practitioners considered extending the use of multipuncture BCG vaccination to schoolchildren in Tower Hamlets. This study was conducted to assess the rate of conversion of the tuberculin test, the acceptability and safety of the device in this age group, and to compare it with the traditional intradermal method.

\section{Methods}

The study was conducted at schools in the London Borough of Tower Hamlets during the 
Table 1 Conversion of the Heaf test

\begin{tabular}{lll}
\hline $\begin{array}{l}\text { Heaf test grade before and } \\
\text { after BCG (test 1-test 2) }\end{array}$ & $\begin{array}{l}\text { Intradermal } \\
\text { method }(n=88)\end{array}$ & $\begin{array}{l}\text { Multipuncture } \\
\text { method }(n=81)\end{array}$ \\
\hline $0-0$ & 1 & 6 \\
$0-1$ & 43 & 45 \\
$0-2$ & 27 & 8 \\
$1-1$ & 5 & 16 \\
$1-2$ & 12 & 6 \\
Total cases of conversion & $82(93.2 \%)$ & $59(72.8 \%)^{\star}$ \\
\hline
\end{tabular}

*Odds ratio 0.2 (95\% CI 0.07 to 0.55$), \chi^{2}=12.6$, p<0.001.

annual BCG vaccination programme. The study was approved by the ethics committee of the East London and City Health Authority. The nature of the study was explained and a written consent form was sent to the children's parents. To ensure consistency two examiners performed all Heaf tests and BCG vaccinations and assessed the results of the tests and the vaccination site. Batch numbers of both types of vaccinations were noted but not taken into account during study analysis.

All children who had not been previously vaccinated and who had no contraindication to tuberculin testing or to BCG vaccination were included. They were tuberculin tested using the Heaf gun. The Heaf test was assessed after seven days and those with grade $0-1$ reactions were included in the study with parental written consent. The children were randomised to receive the BCG either by the intradermal or the multipuncture method.

High strength BCG vaccine with each vial containing 50-250 million colony forming units (Evans Medical) was used for the multipuncture method. ${ }^{6}$ Each vial was constituted with $0.3 \mathrm{ml}$ sodium chloride. Eighteen needle disposable heads set to penetrate to a depth of $2 \mathrm{~mm}$ (Bignal Surgical Instruments) were used to administer the BCG through the skin overlying the left deltoid muscle. A single puncture was applied.

The intradermal vaccine was prepared in the standard way. ${ }^{6}$ Injection of $0.1 \mathrm{ml}$ of the vaccine (Evans Medical) was performed using a $1 \mathrm{ml}$ disposable syringe and 24 gauge needle. Each vial contained 5-25 million colony forming units. The site of injection was the skin overlying the insertion of the left deltoid muscle.

The BCG site was inspected after eight weeks and inflammatory changes, abscess formation, and vesiculation were noted. The transverse diameter of the induration of the response to BCG was measured. At this visit an examiner completed a questionnaire about the child's attitude to immunisation (apprehension about the vaccination) and any pain resulting from the vaccination. The Heaf test was then repeated on the opposite arm to the first test and the results were assessed seven days later by an examiner unaware of the BCG type and the results of the initial Heaf test. The results of the two Heaf tests were compared. A conversion was considered to have occurred if there was at least one grade increment in the second test compared with the first test - that is, from grade 0 to grade 1 , from grade 0 to grade 2 , or from grade 1 to grade 2 .

\section{Results}

One hundred and sixty nine children (83 girls) completed the study. Eighty one of mean age 11.3 years (range 10-14) received the BCG vaccination by the multipuncture method and 88 of mean age 11.4 years (range 10-14) were vaccinated by the intradermal method. The ethnic distribution was similar in both groups.

The Heaf test showed tuberculin conversion in 82 of the 88 children $(93.2 \%)$ receiving the intradermal vaccine compared with 59 of 81 $(72.8 \%)$ of those receiving the multipuncture method (odds ratio $0.2,95 \%$ confidence interval 0.07 to $0.55, \chi^{2}=12.6, p<0.001$ ). Table 1 shows details of the conversion of the Heaf test.

Initially, apprehension about the BCG vaccination was similar in both groups. Children who received the vaccine by the intradermal method experienced pain more often than those who had the multipuncture method. Inflammatory changes, vesiculation, and discharge from the BCG site were also more common in those receiving the intradermal method. A BCG inflammatory response was not visible in $17.3 \%$ of children following use of the multipuncture device. All children who were immunised by the intradermal method had a visible scar. Table 2 shows details of the side effects of the two methods.

There were no significant inter-examiner differences with regard to the conversion rate of the Heaf test for either the multipuncture or the intradermal methods.

\section{Discussion}

This is the first study to compare the efficacy of BCG vaccination and its side effects using the Bignal multipuncture device with the reusable handle and disposable heads. This study is also the first in schoolchildren to evaluate the results of the BCG vaccination by the Heaf test. Previous studies used the increase in diameter of the reaction to the Mantoux test. ${ }^{710}$

Conversion of the Heaf test eight weeks after BCG vaccination was less frequent in those vaccinated by the multipuncture method than

Table 2 Inflammatory changes in the injection site and attitude towards BCG vaccination. Odds ratio refers to the multipuncture method

\begin{tabular}{|c|c|c|c|c|c|}
\hline & $\begin{array}{l}\text { Intradermal method } \\
(n=88)\end{array}$ & $\begin{array}{l}\text { Multipuncture } \\
\text { method }(n=81)\end{array}$ & $\begin{array}{l}\text { Odds ratio (95\% } \\
\text { confidence interval) }\end{array}$ & $\chi^{2}$ & $p$ value \\
\hline Apprehension from injection & $49(55.7 \%)$ & $53(65.4 \%)$ & $0.66(0.34$ to 1.29$)$ & 1.4 & NS \\
\hline Pain & $51(58 \%)$ & $23(28.4 \%)$ & $0.49(0.33$ to 0.72$)$ & 15.0 & $<0.001$ \\
\hline Inflammatory changes & $47(58.0 \%)$ & $10(12.3 \%)$ & $0.23(0.13$ to 0.43$)$ & 30.0 & $<0.001$ \\
\hline Vesiculation & $24(27.3 \%)$ & $0(0 \%)$ & -夫 & 25.6 & $<0.001$ \\
\hline Discharge from BCG site & $13(14.8 \%)$ & $1(1.2 \%)$ & $0.8(0.01$ to 0.62$)$ & 10.1 & $<0.002$ \\
\hline Scar visible & $88(100 \%)$ & $67(82.7 \%)$ & $0.83(0.75$ to 0.91$)$ & 16.5 & $<0.001$ \\
\hline
\end{tabular}

^Odds ratio could not be calculated due to the zero value. 
by the intradermal method. This may be due to the fact that the dose of BCG delivered to the dermal layer of the skin using the 18 needle head was not sufficient. Previous studies have indicated that a $2 \times 20$ puncture resulted in a higher conversion rate than a $1 \times 20$ puncture. ${ }^{9}$ The manufacturers assert that a device with 40 heads is not practical as it requires an unacceptably high pressure to release the needles (Colin Bignal, personal communication).

Cundall et $a \bar{l}$ found that, in infants and small children, both methods of vaccination gave similar conversion rates of the tuberculin test. The rate of conversion, however, differed significantly among doctors using the intradermal method but not the multipuncture method. In our study, which was conducted on older children, there was no difference in the conversion rate between examiners with either method of vaccination.

The multipuncture method resulted in fewer undesirable side effects than the intradermal method and, because it was easier to administer, it would be advantageous in the school setting. The BCG scar was also less visible than with the intradermal method. This is an advantage from the aesthetic aspect but means that there is no visible record of immunisation. Consequently, there would be a greater onus on accurate record keeping and ensuring that students have their own immunisation records.

Conversion of the Heaf test is a crude method of measuring BCG efficacy as it mainly measures change in hypersensitivity to the protein part of the tuberculosis bacilli. Failure of conversion following BCG administration may not necessarily mean a failure to protect against tuberculosis. In a meta-analysis of previous studies of the efficacy of BCG in the prevention of tuberculosis the type of vaccination (multipuncture versus intradermal) did not explain the difference in efficacy between studies. ${ }^{11}$ Conversion of the Heaf test was used in the current study, as in previous studies, ${ }^{7-11}$ as an indirect method of measuring the efficacy of BCG vaccination.

Although this study did not show equivalent conversion of the tuberculin test following BCG immunisation with the multipuncture method compared with the traditional intradermal method, we believe that there are many advantages to the multipuncture method. It is easy to use, requires less skill and training, and is less time consuming. We therefore recommend further work to ascertain the correct dosage of BCG to be carried by the multipuncture device to ensure an improved tuberculin conversion rate.

The authors gratefully acknowledge the considerable assistance provided by Marion Whyte, Dr Rex Obeng, and Liz Swann and thank Dr F Festenstein for her help during the preparation of this manuscript.

The study was self initiated and was not funded by any organisation.

Conflict of interest: none.

1 Tuberculosis Vaccines Clinical Trials Committee of the Medical Research Council. BCG and vole bacillus vaccines in the prevention of tuberculosis in adolescents. BMF in the prevention $1956 ; 1: 413-27$

2 Springett VH, Derbyshire JH, Nunn AJ, et al. Changes in tuberculosis notification rates in the white ethnic group in
England and Wales between 1953 and 1983. F Epidemiol England and Wales between 1953

3 Sutherland I, Springett VH. The effects of the scheme for BCG vaccination of schoolchildren in England and Wales and the consequences of discontinuing the scheme at various dates. F Epidemiol Community Health 1989;43:15-24.

4 Packe GE, Innes JA. Protective effect of BCG vaccination in infant Asians: a case-control study. Arch Dis Child 1988;63: $277-81$

5 Cundall DB, Ashelford DJ, Pearson SB. BCG immunisation of infants by percutaneous multiple puncture. BMf 1988;279:1173-4.

6 Department of Health. BCG immunisation. In: Immunisation against infectious disease. London: HMSO, 1996: 236.

7 Griffith MI, Brindle TW, Gordon EH, et al. Multiplepuncture vaccination with freeze-dried BCG vaccine in puncture vaccination with freeze-dri
schoolchildren. BMF 1961;1:536-40.

8 Griffith AH, Kinsley BJ, Anderson DJ. A comparison Griffith AH, Kinsley BJ, Anderson DJ. A comparison
between multipuncture and intradermal methods of BCG between multipuncture and intrader

9 Research Committee of the British Tuberculosis Association. BCG vaccination by multi-puncture: third report. Tubercle 1965;46:111-20

10 Research Committee of the British Thoracic and Tubercuosis Association. BCG vaccination by multiple-puncture: fourth report. Tubercle 1971;52:19-30.

11 Colditz GA, Brewer TF, Barkey CS, et al. Efficacy of BCG vaccine in the prevention of tuberculosis. Meta-analysis of the published literature. $f A M A$ 1994;271:698-702. 